\title{
Skin Development during the Film Formation of Waterborne Acrylic Pressure-Sensitive Adhesives containing Tackifying Resin
}

\author{
J. Mallégol, ${ }^{1+}$ G. Bennett, ${ }^{1}$ O. Dupont,${ }^{2}$ P. J. McDonald ${ }^{1}$ and J. L. Keddie ${ }^{1 *}$ \\ 1. Department of Physics, School of Electronics and Physical Sciences, University of \\ Surrey, Guildford, Surrey GU2 7XH, United Kingdom
}

2. Cytec Surface Specialties, 33 Anderlechtstraat, Drogenbos B1620, Belgium

\begin{abstract}
Tackifying resins (TR) are often used to improve the adhesive properties of waterborne pressure-sensitive adhesives (PSAs) derived from latex dispersions. There is a large gap in the understanding of how, and to what extent, the film formation mechanism of PSAs is altered by the addition of TR. Herein, magnetic resonance profiling experiments show that the addition of TR to an acrylic latex creates a coalesced surface layer or "skin" that traps water beneath it. Atomic force microscopy of the PSA surfaces supports this conclusion. In the absence of the TR, particles at the surface do not coalesce but are separated by a second phase composed of surfactant and other species with low molecular weight. The function of the TR is complex.. According to dynamic mechanical analysis, the TR increases the glass transition temperature of the polymer and decreases its molecular mobility at high frequencies. On the other hand, the TR increases the molecular mobility at lower frequencies and thereby promotes the interdiffusion of latex particles to create a skin layer.In turn, the skin layer is a barrier that prevents the exudation of surfactant to the surface. The TR probably enhances the coalescence of the latex particles by
\end{abstract}

\footnotetext{
${ }^{+}$Current address: Arcelor Research Liège SCRL, Bd. de Colonster, B57, B-4000 Liège, Belgium
} 
increasing the compatibility between the acrylic copolymer and the solids in the serum phase.

Running Title: Film Formation of Tackified Waterborne Adhesives

Key words: tackifier; latex; film formation; atomic force microscopy; magnetic resonance profiling; coalescence

\section{Introduction}

Pressure-sensitive adhesives (PSAs) constitute a distinct category of adhesives that instantly wet and firmly adhere to a variety of dissimilar surfaces, when applied with only light pressure, without activation by water, heat or solvent.[1] A major contribution to the energy that is required to debond two surfaces joined by a PSA is from its viscoelastic energy dissipation.[2,3] Recent work by Brown and co-workers [4] has vividly demonstrated the influence of polymer mobility on the adhesion force between solids and polymers. The adhesive performance of a PSA depends strongly on the balance of viscoelastic properties, and hence a considerable amount of research has aimed to correlate the two. $[1,5,6,7]$

PSAs are often manufactured from acrylic ester copolymers that have a glass transition temperature, $T_{\mathrm{g}}$, as low as $-60^{\circ} \mathrm{C}$. These polymers are inherently tacky without any additional compounding, and their adhesive properties can be modified through a variation of the copolymer composition. It is a common practice, however, to incorporate a tackifying resin (TR), also known as a tackifier, in acrylic formulations to enhance their properties further.[6,8,9] A TR is usually a bulky, low molecular weight molecule, such as n-butyl ester of abietic acid [10] or

\footnotetext{
*Corresponding author: j.keddie@surrey.ac.uk
} 
pentaerythritol rosin ester derived from abietic acid.[11] The properties of a PSA can be finely tuned through the addition of an appropriate TR.[12] The effects of tackifiers on viscoelasticity and adhesion have been thoroughly studied ${ }^{[13,14,15,16]}$. The effect of a compatible TR on polymer viscoelastic properties is twofold. First, the elastic modulus E', at the temperature of the PSA's use, is lowered. Second, blending with a compatible tackifier causes a shift of the glass transition temperature $\left(T_{\mathrm{g}}\right)$ to a higher value.[8] The lower $E^{\prime}$ promotes polymer flow and bond formation and, coupled with the higher $T_{\mathrm{g}}$, enhances the resistance to bond rupture.[17] In terms of the strain rate, a tackified PSA is stiffer at high strain rates, such as during debonding, but it flows more easily at low strain rates, such as when wetting a substrate.[8]

In acrylic PSAs, the most noticeable effect of the addition of TR is the enhancement of tack and peel strengths to substrates with a low surface energy, such as polyolefines and carton board.[6] Problems presented by the use of TR in acrylic PSAs are a lower resistance to the ageing of TR/PSA mixtures and a lower shear holding power.[6] Although the two phases can be soluble in a common solvent, when the solvent leaves the PSA, phase separation can occur. Tackified PSAs can also undergo slow phase separation and surface segregation, resulting in a change in the adhesive and mechanical properties.[10] Hence, the degree of compatibility between the polymer and TR is a very important factor in formulating a PSA. This fact explains the great interest in TR miscibility with polymers and hence the large number of publications on the miscibility in such systems.[18,19,20,21,22,23,24] 
Tighter environmental regulations in the production of PSAs have led to a shift away from solvent-cast formulations to aqueous dispersions of polymer colloids, i.e. latex. Waterborne PSA technology has increasingly more often been a subject of research, as indicated in a recent review article.[25] Generally it has been found that the performance of waterborne acrylic PSAs is inferior to that of their solvent-based analogues. They exhibit lower water resistance,[26] a tendency to whiten in moist atmospheres, and lower tack, adhesion and shear strengths.[6,27,28,29,30] This relatively poor performance of waterborne PSAs has been correlated with a heterogeneous film morphology[27] and with the discontinuity of the molecular network structure[30] of dry films. The distribution and migration of small molecules, especially surfactants,[27,29,31] has also been correlated with poor waterborne adhesive performance. In our previous research,[32,33,34] we have determined the morphology of waterborne PSAs as a first step in improving their performance.

In formulating waterborne PSAs, the use of TR dispersions in water is preferred for obvious reasons. The inferiority of waterborne PSA shear strength, however, is even greater when waterborne, acrylic PSAs are compounded with waterborne dispersions of TR.[9] Furthermore, in waterborne, tackified PSAs, an additional level of complexity is added by the requirement for compatibility of latex and tackifier emulsifiers with each other and with the polymer.[35]

There has been only limited published research on the characteristics of waterborne, tackified PSAs. [9,17,35,36] The work of Tobing and Klein[36] is particularly significant because it considers the mechanism by which tackifiers improve waterborne PSA performance. These authors commented, however, that the 
development of tackified PSA technology was being limited by a poor understanding of the film formation process. The present work was motivated by this knowledge gap and by an appreciation of the importance of film formation in determining waterborne PSA properties.[25]

In our previous research,[33] we found with magnetic resonance (MR) profiling that during the drying of an acrylic waterborne PSA film, a water concentration gradient develops. There was less water near the interface with the atmosphere, however the water level never receded from this interface. Unlike the drying of silicone[37] and alkyd[38] emulsions, studied elsewhere, there was no evidence for a dry surface layer or "skin". Atomic force microscopy (AFM) images of dry PSA surfaces revealed that the particles were not coalesced but were separated from their neighbors by a liquidlike medium. With complementary use of Rutherford backscattering spectrometry (RBS), we deduced that surfactant (possibly in addition to other water-soluble species) was present in excess at the surface, where it stabilizes the particles against coalescence. We speculated that because particles did not coalesce during this stage of drying, the distribution of surfactant (and other water-soluble species) along the particle/particle boundaries was enabled.

In the present work, we consider the drying and film formation mechanisms in tackified, waterborne acrylic PSAs. We provide the first report of how the presence of a waterborne TR modifies the water concentration profiles and particle coalescence in a waterborne acrylic PSA. We show that the TR has a major impact. There are no thorough studies of phase stability and ageing in tackified, waterborne acrylic PSAs. Therefore, this issue was also investigated in the current work. 


\section{Experimental Materials and Methods}

\section{Materials}

A model acrylic PSA latex (referred to in previous publications [32,33] as PSA A) was investigated. This latex, with a solids content of $60 \mathrm{wt} . \%$, has a bimodal particle size distribution (weight-averaged particle sizes of $180 \mathrm{~nm}$ and $350 \mathrm{~nm}$ determined by dynamic light scattering (Nicomp 370 particle sizing systems)) and a solids content of $60 \mathrm{wt} . \%$. A random copolymer that makes up the latex particles consists mainly of 2 ethylhexyl acrylate, an acrylic ester that yields a soft and tacky material with a low glass transition temperature $\left(\mathrm{T}_{\mathrm{g}}\right)$, with additions of methyl methacrylate and polar monomers (acrylic acid and methacrylic acid). DSC analysis of the copolymer obtained a $\mathrm{T}_{\mathrm{g}}$ of $-45^{\circ} \mathrm{C}$. The latex was prepared by standard techniques of semi-batch emulsion polymerization. Dissolution of the latex polymer in organic solvent has revealed a low insoluble fraction, which indicates the presence of polymer molecules with very high molecular weight and/or a partially cross-linked network. The looptack strength of the PSA film, determined according to Finat Test Method No. 9 (FTM9), is $12.8 \mathrm{~N} /$ inch (on stainless steel substrate).

The TR is a stabilized rosin ester (Tacolyn ${ }^{\circledR} 3189$ from Eastman Chemical, Kingsport, Tenn., USA) dispersed in water at a solids content of $50 \mathrm{wt} . \%$ and with a weightaverage particle size of $220 \mathrm{~nm}$ According to DSC, the $T_{\mathrm{g}}$ of the dry TR is $29^{\circ} \mathrm{C}$, but this value is as low as $20^{\circ} \mathrm{C}$ when it is not fully dried, as a result of plasticization by water. The softening point, determined by the Hercules drop method, is $70{ }^{\circ} \mathrm{C}$. The latex was blended with the TR dispersions at various concentrations: $1 \%, 5 \%, 10 \%$, 
$18 \%, 25 \%, 33 \%, 41 \%, 50 \%$, and $75 \%$ tackifier (by weight). The blended dispersions were stirred for several hours to ensure complete mixing.

\section{Dynamic Mechanical Analysis}

The dynamic tensile moduli (E' and E') of the acrylic PSA and the tackified PSA films were determined by dynamical mechanical analysis in tensile deformation using a 2980 Dynamic Mechanical Analyzer (TA Instruments, New Castle, USA). A strain oscillation with an amplitude of $0.1 \%$ was applied at a frequency of $1 \mathrm{~Hz}$. The temperature was increased from -60 to $120{ }^{\circ} \mathrm{C}$ at a heating rate of $3{ }^{\circ} \mathrm{C} / \mathrm{min}$. The linearity condition was verified at selected temperatures.

Free-standing PSA films ( $1 \mathrm{~mm}$ thick) were prepared by de-laminating films that had been cast on release paper, dried for $48 \mathrm{hr}$. at ambient temperature, and then heated for $16 \mathrm{hr}$. at $50{ }^{\circ} \mathrm{C}$. Before analysis, the films were submitted to an additional heating at $110{ }^{\circ} \mathrm{C}$ for $2 \mathrm{hr}$. Lateral dimensions of the rectangular film samples were $10 \mathrm{~mm} \mathrm{x}$ $8 \mathrm{~mm}$.

\section{Atomic force microscopy of PSA surfaces}

Films were cast onto silicone-coated paper release liners $(30 \mathrm{~cm}$ x $20 \mathrm{~cm})$ using a 40 $\mu \mathrm{m}$ hand-held bar coater. The films were dried under laminar air flow for $3 \mathrm{~min}$. on heated plates at $60{ }^{\circ} \mathrm{C}$ in a controlled humidity chamber maintaining a relative humidity of $40 \%$. The dried films for AFM analysis were about $20 \mu \mathrm{m}$ thick.

Small pieces $(1 \mathrm{~cm} \times 1 \mathrm{~cm})$ of the cast PSA were cut from the large-area films and were analyzed with an atomic force microscope (Nanoscope IIIa, Digital Instruments, 
Santa Barbara, CA, USA) within three hours of casting. All measurements used a silicon cantilever (NT-MDT, Moscow, Russia) equipped with an ultrasharp, conical silicon tip having a radius of curvature of about $10 \mathrm{~nm}$. The nominal resonant frequency $f_{o}$ of the cantilever is $320 \mathrm{kHz}$ and its spring constant $k$ is $48 \mathrm{~N} / \mathrm{m}$.

AFM analysis was performed on the original interface with air. Images were recorded simultaneously in the topographic (height) mode and in the phase mode, with scan sizes ranging from $5 \mu \mathrm{m}$ to $30 \mu \mathrm{m}$. The optimum method for obtaining images of latex PSA surfaces has been reported previously.[32] Parameters needed to describe the tapping conditions are the "free" amplitude $A_{o}$ and the setpoint value $d_{s p}$.

The high tack of the PSA surface makes it necessary to use high tapping amplitudes to impart enough energy to the tip to "pull off" of the adhesive surface. A high setpoint ratio $\left(d_{\mathrm{sp}} / A_{\mathrm{o}}\right)$ is required to minimise indentation of the tip and thereby to reduce deformation of the soft surface. To obtain values of $A_{o}$ and $d_{s p}$ in metric units, a systematic calibration of the cantilever was obtained from amplitude-distance curves on a clean silicon wafer, assuming no deformation of the silicon surface and no bending of the cantilever during tapping.[39]

All AFM images presented here were obtained with very similar tapping conditions. Typically, $d_{s p}$ was between 90 and $100 \mathrm{~nm}$ for all measurements. $A_{o}$ was typically 20 $\mathrm{nm}$ above the $d_{s p}$ for the original air interface of the PSAs and 30 to $40 \mathrm{~nm}$ above the $d_{s p}$ for the face delaminated from the release liner. These tapping conditions ensure that the indentation of the AFM tip into the PSA surface is small enough to avoid significant distortion of the morphology. It is, however, important to realise that the 
the AFM tip does indent into the polymer surface, as shown previously, but that the structure is not permanently altered.[32,34]

\section{NMR Spectroscopy}

${ }^{1} \mathrm{H}$ NMR spin-spin relaxation time $\left(\mathrm{T}_{2}\right)$ distributions were measured for dried films with varying concentrations of TR on a low resolution, $20 \mathrm{MHz}$ spectrometer (Maran, Oxford Instruments, Abingdon, Oxon., UK). Specifically, free induction decay (FID) and Carr-Purcell-Meiboom-Gill (CPMG) echo train signals were acquired. $\mathrm{T}_{2}$ is a measure of molecular mobility; a higher value indicates greater mobility.

The free induction decays were recorded with a sampling rate of $2 \mathrm{MHz}$ following a short $90^{\circ}$ excitation pulse of $3.9 \mu$ s duration, enabling the observation of faster relaxing components. However, the instrument's dead time of $c a .25 \mu$ s prevented the very fastest components of the dried TR from being detected. The CPMG trains were used to observe the slowly relaxing components better. The basic $90^{\circ}-180^{\circ}$ pulse gap was $64 \mu$ s. Alternate echoes were recorded out to $16.4 \mathrm{~ms}$. In both experiments, a total of 64 averages was collected at a repetition interval of $2 \mathrm{~s}$. To prepare samples, aqueous dispersions were cast on silicone-coated paper (using an $80 \mu \mathrm{m}$ bar coater), dried at $60{ }^{\circ} \mathrm{C}$ for 10 min., de-laminated from the surface, and then rolled and inserted into glass NMR tubes $\left(1 \mathrm{~cm}\right.$ diameter) for analysis at $23^{\circ} \mathrm{C}$. The samples were all prepared on the same day under identical conditions and analysed approximately $3 \mathrm{hr}$ after casting.

An additional experiment was carried out to examine the rate of mixing of the TR and PSA. Two films of pure TR and pure PSA were cast separately on silicone paper. 
After they were dry, they were pressed together face-to-face, then peeled from the silicone-coated paper substrates, and finally inserted into an NMR tube for analysis. Initially the two substances are completely separate, and there is no molecular interaction, except perhaps at the interface. The FID was obtained at various times after bringing the two materials into contact.

\section{MR Profiling}

A small permanent magnet, which was designed specifically for obtaining magnetic resonance profiles of ${ }^{1} \mathrm{H}$ in planar films, was used to probe the water concentration in drying latex layers in the direction normal to the substrate. The details of this magnet, called Gradient At Right-angles to the Field (or GARField), have been reported previously.[40,41] In the experiments performed here, samples were placed in the magnet at a position corresponding to a magnetic field strength of $0.7 \mathrm{~T}$ and a field gradient strength of $17.5 \mathrm{~T} \mathrm{~m}^{-1}$. In experiments, latex films were cast onto clean glass coverslips $(2 \mathrm{~cm} \times 2 \mathrm{~cm}$ ) using either a $120 \mu \mathrm{m}$ or a $250 \mu \mathrm{m}$ applicator. Immediately after casting, the film was placed in the magnet. MR profiling was commenced with the sample in the open atmosphere at an average temperature of $23{ }^{\circ} \mathrm{C}$ within the instrument. Signals were obtained using a quadrature echo sequence[42]: $90_{\mathrm{x}}-\tau-$ $\left(90_{\mathrm{y}}-\tau \text { - echo }-\tau-\right)_{\mathrm{n}}$ for $\mathrm{n}=32$ echoes and a pulse gap of $\tau=95.0 \mu \mathrm{s}$. To obtain a profile, the echoes were Fourier-transformed and then summed, thus giving the NMR signal intensity profile as a function of depth with a pixel resolution of $8.8 \mu \mathrm{m}$. Profiles were normalized by an elastomer standard in order to correct for the sensitivity decline over the film thickness.

\section{Results and Discussion}




\section{Effect of Tackifier on Mechanical Response}

The dynamic mechanical analysis shows that the addition of tackifier has a significant effect on the dynamic tensile moduli of the PSA. In Figure 1 the storage modulus (E') and $\tan \delta=E^{\prime} / E^{\prime}$ of the neat acrylic latex is compared to the same latex containing 25 wt. $\%$ TR. There are several differences. The addition of the tackifier increases the observed $\mathrm{T}_{\mathrm{g}}$ from -20 to $-5^{\circ} \mathrm{C}$. At temperatures near the glass transition temperature, E' and $\tan \delta$ of the tackified PSA are higher. Above $\mathrm{T}_{\mathrm{g}}$, a lower E' is found in the tackified PSA, indicating that the polymer can flow more readily to achieve good wetting of a substrate. These results can be extrapolated to predict that the tackified PSA is stiffer and more energy dissipative at high strain rates, such as during debonding, but it flows more easily at low strain rates, such as when wetting a substrate.[8]. As a result of these various effects, the addition of TR will improve the looptack and peel strengths of the PSA. Investigation of the film formation process was carried out to determine how these properties were obtained.

\section{Film Formation Mechanisms}

There have been a few studies of waterborne PSA film formation,[25] but there is scarce knowledge of the film formation of waterborne tackifiers, even though problems with adhesive performance have been blamed on this lack of understanding. [30] To address this gap in the literature, the film formation of the neat TR dispersion and tackified acrylic PSA films were observed using AFM.

Even though the $T_{\mathrm{g}}$ of the dry $\operatorname{TR}\left(29^{\circ} \mathrm{C}\right)$ was above room temperature $\left(23^{\circ} \mathrm{C}\right)$, it was found that a smooth, transparent film could be formed by casting the TR dispersions. This observation can be explained by the plasticization of the TR by water. 
Furthermore, rosins typically have a low molecular weight and have been found elsewhere to have a high self-diffusion coefficient $\left(\mathrm{ca} \cdot 10^{-8} \mathrm{~cm}^{2} \mathrm{~s}^{-1}\right)$ associated with high molecular mobility.[43]

An AFM image obtained 20 min. after casting shows that the tackifier particles at the film surface are between about 50 and $200 \mathrm{~nm}$ in diameter (Figure 2a). After further drying, the particle boundaries have lost their definition and appear to have fused together. Whereas in latex films, the particles are often deformed from their spherical shape to create rhomboid dodecahedra and other geometries with flat faces,[44] the TR particles remain as spheres at 60 min. (Figure 2b). There is no evidence that they have been deformed elastically. Within two hours, a smooth surface emerges, with no topographic features whatsoever (Figure 2c). There is no evidence for a second phase in the substance. No information can be obtained about the sub-surface, of course, from this type of analysis.

Further AFM analysis investigated the effects of blending the TR with the acrylic latex. As found in our previous study,[33] in the acrylic latex (without any TR addition) there is a second phase (lighter areas in the phase contrast image in Figure 3a) that surrounds each particle. Particle boundaries are sharply defined. This second phase, which consists of surfactant and other low molecular weight, water-soluble species, inhibits particle coalescence.

When only 5 wt.\% TR is blended with the latex, blurring of the particle boundaries is evident in the AFM images (Figure 3b). Nevertheless, there is still some evidence for a second phase in the phase contrast image, albeit less pronounced than in the neat 
latex. Individual particles of the TR cannot be identified in the image with any level of confidence. With $10 \mathrm{wt} . \% \mathrm{TR}$, the surface becomes even more homogeneous, and when there is $25 \mathrm{wt} . \%$ TR in the PSA blend, the particle boundaries have practically disappeared (Figures 3c and 3d). A completely homogeneous surface is obtained with $50 \mathrm{wt} . \%$ TR in the PSA formulation (Figure 3e). Just as was observed at the surface of the neat TR, there are no topographical features and no evidence for a second phase. This is a surprising result, because the particles in this acrylic latex have been found previously to retain their identity even when heated or subjected to shear stress.[34] A second phase is always observed at the surface of the neat acrylic latex, whereas with the addition of TR, this phase is no longer apparent.

This is an intriguing result. Even though the TR has a higher $\mathrm{T}_{\mathrm{g}}$ than the acrylic, the addition of the TR leads to better particle coalescence. Conventionally, better coalescence is achieved through the addition of solvents (or coalescing aids) that decrease the polymer's $\mathrm{T}_{\mathrm{g}}$ and enhance the polymer mobility. [45] The DMA data reveal that the addition of the TR decreases E' (and increases molecular mobility) at higher temperatures (and lower frequencies). The greater molecular mobility at lower frequencies and over longer times should correlate with a shorter polymer reptation time and therefore faster interdiffusion between particles. Hence, the observed enhanced interdiffusion rates between the particles is consistent with the DMA results.

Further experiments were conducted to understand how this PSA structure emerges. In particular, techniques of NMR spectroscopy were employed to determine the miscibility of the acrylic and tackifier. Figure 4 shows the front end of magnetization 
decay curves constructed from the FID and CPMG experiments. To aid comparison, the decays have been normalised to unit amplitude, using analytical fits to the data. The decay profile of the pure acrylic cannot be described as a single exponential curve. It can be described with a somewhat broad distribution of relaxation time constants centered at a time constant, $\mathrm{T}_{2}$, of $500 \mu \mathrm{s}$. The distribution of relaxation times reflects the slightly varying local environments of the ${ }^{1} \mathrm{H}$ in the acrylic, such as the distance from a crosslink point and the monomer composition.

The decay of the pure TR, by contrast, is best described by two relaxation time constants, with one being significantly shorter than the other. The short component is described by a $\mathrm{T}_{2}$ of $25 \mu \mathrm{s}$. The distribution of the longer component $\mathrm{T}_{2}$ values are best described as being bi-modal. One of the relaxations in the longer component is described with a $\mathrm{T}_{2}$ of $340 \mu \mathrm{s}$. It is likely that the other relaxation in the longer component - that with a smaller amplitude and a higher $\mathrm{T}_{2}$ value - is due to residual water trapped in the resin film. This hypothesis is supported by our observation that the longer component decay becomes more uni-modal when a TR film is aged under ambient conditions for several days. Figure 4 shows the data from a freshly prepared TR film.

Figure 4 reveals that as the TR is blended with the neat acrylic at greater concentrations, the decay profiles are increasingly and more obviously multiexponential in character. Specifically, a short (rapidly-decaying) component develops, and it amplitude increases at the expense of the long component. This component is attributed to the TR. The longer $\mathrm{T}_{2}$ of the acrylic component decreases from $500 \mu$ s to $340 \mu$ s in the pure TR. 
Two observations in the NMR data provide evidence that the acrylic and TR are blending at the molecularlevel (rather than the particle level). One observation is that the $T_{2}$ values of both the long and short components decrease with increasing tackifier concentration. If there were not any mixing at the molecular level, then the $\mathrm{T}_{2}$ values would be constant. The second observation is that the decay of an intermediate composition, such as the $50 \mathrm{wt} . \% \mathrm{TR} / \mathrm{acrylic}$ blend, cannot be created by a linear sum of decays for the neat TR and the acrylic. The acrylic chain mobility is increasingly hindered by the tackifier. Hence, the slow decay attributed to the acrylic is lost in the blends.

The mixing and interaction between the acrylic and TR were followed over time using the FID measurements as a probe. Films of the acrylic and TR with equal thickness were stuck together face-to-face and then placed into an NMR tube for analysis. Initially the two products are completely separated and no molecular mixing has occurred. The initial FID curve should therefore be equivalent to the average of that for the two substances. The first FID measurement was obtained $20 \mathrm{~min}$. after contact was made between the films, and it is shown in Figure 5 (Curve B). This experimental FID is compared to the average of the two substances (Curve A). The faster decay for the bilayer sample suggests that there already is some molecular blending.

Over time up to 260 hours, the FIDs (Curves C, D and E) gradually approach that which was obtained from a dried PSA that was cast from a 1:1 blend of acrylic latex and TR (Curve F). The increase in the decay rate of the FID as contact time is 
increased is attributed to interdiffusion between the acrylic and the TR. The decay is fastest in the dried, cast film, which means that it has the highest level of mixing between the acrylic and TR. This result can be understood by considering that in the colloidal film, the acrylic and TR are mixed on the size scale of hundreds of nm, and so the diffusion distances are very short. In the bilayer, on the other hand, mixing is incomplete, even at relatively long times, owing to the longer diffusion distances. The TR and the acrylic are shown to be miscible, as the FID for the blend is distinctly different than for the individual components.

In the literature, tackifiers are often referred to as a "solid solvent" for polymers used in PSAs. $[8,17,35]$ Although a solid at room temperature, a tackifier can diffuse into a polymer. Whereas a liquid solvent lowers the $T_{\mathrm{g}}$ of a polymer, a tackifier increases it. Our measurements of $T_{2}$ are consistent with this description. The dry TR and the acrylic are miscible over the entire range of mixtures. At temperatures near its $T_{\mathrm{g}}$, the TR is able to interdiffuse with the acrylic.

In summary of this section, the combination of AFM and NMR analysis has revealed that miscibility between the acrylic and the TR has enabled the formation of a fully coalsesced layer or "skin" at the PSA surface.

\section{Drying mechanism}

Looking ahead to the GARField MR profiling measurements, ${ }^{1} \mathrm{H}$ in mobile environments, such as water, gives a strong signal at the GARField first echo measurement time of $c a .200 \mu \mathrm{s}$. The acrylic gives an intermediate signal, and the TR yields no signal. MR profiling was used to gain insight into the mechanism of latex 
drying as TR was added. Data obtained during the entire course of drying of a neat acrylic latex film are presented in Figure 6.

The NMR signal plotted in the profiles is dependent upon both the concentration and mobility of ${ }^{1} \mathrm{H}$ in the latex film, as explained in detail elsewhere.[38] The top surface (air interface) is represented on the right side of the profile, and the substrate interface is at the left. Two discrete stages of drying are apparent. In the first stage (up to about 22 min. of drying time), the concentration of water is apparently uniform with depth from the surface. The thickness of the film decreases at a constant rate as water evaporates. In the second stage (after $22 \mathrm{~min}$.), the rate of thickness decrease slows sharply. Non-uniformity in the water concentration develops, as the water concentration near the film surface decreases. Throughout the stage, the water level is pinned at the film surface, probably by capillary pressure. After 73 min. of drying, the water concentration is seen to increase approximately linearly with depth into the film surface. Thereafter, the concentration gradient remains roughly linear with depth, but it becomes more shallow, and the water concentration near the substrate decreases.

It is important to observe that there is no evidence for a dry, coalesced surface layer at any point in the second stage of drying. On the contrary, there is evidence for a water pathway extending throughout the film depth. As the solubility of water in the latex is negligible, the profiles provide strong evidence that there is not significant particle coalescence during the drying process. Notice also that the NMR signal detected after 218 min. of drying is attributed to the ${ }^{1} \mathrm{H}$ in the acrylic. The $\mathrm{T}_{2}$ measurements, 
presented earlier, indicate that the ${ }^{1} \mathrm{H}$ in the acrylic has sufficient molecular mobility to provide a signal.

With the parameters employed for MR profiling, it is not expected that an NMR signal will be obtained from the TR, because of its lower $\mathrm{T}_{2}$ values (with the dominant one being at $25 \mu \mathrm{s})$. The signal from the dry PSA is expected to increase as the fraction of acrylic increases. This expectation is consistent with the MR profiles presented in Figure 7. For clarity of presentation, only the second stage of drying is shown. As TR is added to the PSA, there is progressively a lower signal from the fully-dried material. With 25 wt.\% TR there is a signal from the PSA (acrylic/TR blend) but at 50 wt.\% TR, there is no signal from the dry PSA, because TR has inhibited the molecular motion of the acrylic molecules.

The water distribution during the drying of the PSA with a TR concentration of 10 wt.\% is very similar to that in the neat acrylic latex. At 25 wt.\% TR, however, an important difference in the profiles is observed. At a drying time of $247 \mathrm{~min}$, it is apparent that the water level, indicated by the arrow, has receded from the film surface of the $200 \mu \mathrm{m}$ thick PSA. Unlike the case of the neat latex, there is no longer a linear gradient in signal increasing from the top to the bottom of the PSA. At a time of 326 min., there is a thicker layer near the surface, and there is apparently very little water in this surface layer. These profiles indicate that a skin has formed on the PSA. It takes more than $600 \mathrm{~min}$. for the signal from the water to disappear, showing that the drying is about three times slower than in the non-tackified acrylic latex. 
At higher fractions of TR, the profiles progressively display this trend. A surface layer with no NMR signal, presumed to be fully dry, develops at the surface, and it increases in thickness over time. With higher fractions of TR, the step in the water concentration at the boundary between the skin layer and the underlying wet region becomes sharper.

Clearly, the addition of TR has a pronounced effect on the drying mechanism. The water distribution in the neat acrylic latex is consistent with capillary pressure pinning water at the PSA surface. In the tackified latex, there is skin formation. The AFM images of the tackified PSA surfaces (Figure 3) revealed good particle coalescence at the surface. This observation, along with the observed water distributions, is consistent with film formation by wet sintering.[46] The particles are being deformed in the presence of the aqueous phase.

As pointed out elsewhere [46,47], when there is skin formation, there is a high likelihood that water will be trapped beneath the surface. Drying rates will be impeded as water diffuses through the skin layer rather than flowing along the particle boundaries and through interparticle voids. The observed drying times support this notion of a slowdown in drying as a result of skin formation. Table 1 lists the approximate drying times as a function of TR concentration. The total time for water to be lost from the PSA increases as TR is added. The TR dries more than six times more slowly than the neat acrylic latex, which is consistent with skin formation in the former. 
In previous work, we speculated that the drying mechanism facilitated the transport of surfactant to the surface. It would be expected, therefore, that with a concentration of $25 \mathrm{wt} . \%$ or more TR in the PSA, when skin formation is observed, there would be less surfactant at the air surface, because its transport would be impeded. RBS was used to search for surfactant near the surface, by the method described elsewhere.[33,48] S and $\mathrm{K}$ are both found in the surfactants used in the latex synthesis. According to our RBS analysis, there was an excess of $\mathrm{S}$ and $\mathrm{K}$ at the acrylic latex surface. In a PSA containing 25 wt.\% TR, however, no surface excess was observed. Thus, it appears that a sealed skin layer prevented the transport of the surfactants to the surface during the later stage of drying.

In summary, the combination of AFM and NMR analysis has revealed that miscibility between the acrylic and TR has enabled the formation of a fully coalesced layer at the PSA surface. The formation of a continuous layer is allowed because the TR is compatible with the solids in the latex serum (such as surfactants) as well as with the acrylic. The TR thus acts like a "compatibilizing phase" between latex particles and the latex serum solids.

Acrylic/TR Interdiffusion and Ageing of PSAs

Owing to the miscibility of the acrylic and TR, phase separation is not expected in the PSA. On the other hand, further interdiffusion between the TR and acrylic could produce an evolution of the PSA morphology. AFM experiments were conducted over time to explore this possibility.

The AFM images of the neat acrylic latex in Figures 8a-c, obtained at 2 hrs, 3 weeks, and 7 weeks after film casting, show some changes in the structure. There is some 
fusion of particles at their points of contact. The solids in the serum phase (such as the surfactants and water-soluble polymers) will be referred to hereafter as the "serum solid phase." Whereas at the freshly-cast film surface, the serum solid phase fully surrounds each particle, at the aged surfaces, this phase is not fully continuous. There is some coarsening of the structure and an increased level of contact between the acrylic particles.

This evolving structure differs greatly from the surface of a PSA containing 1 wt.\% TR. Even with this low concentration of TR, particle boundaries are not as distinctlydefined at the freshly-cast surface (Figure 8d). Over time, the particles appear to dissolve into each other, with their boundaries becoming blurred. This apparent "dissolution" is in contrast to the fusion at specific points of contact seen in the neat acrylic. After ageing one week, the particle boundaries are no longer apparent, but the surface is heterogeneous.

It is not possible to distinguish between the TR and the serum solid phase in the images. The TR might be acting as a compatibilizer between the serum solids (e.g. surfactants) and the latex particles. By blending with the serum solids, the TR is able to form a continuous phase. Miscibility with the acrylic is retained, so that this continuous phase does not become trapped at the particle interfaces but can interdiffuse with the acrylic. The hydrophilicity of the surfactant might increase water solubility of the acrylic. Water could then plasticize the acrylic.

These contrasting mechanisms are illustrated schematically in Figure 9. In the acrylic latex, the latex serum phase (containing surfactants) stabilizes the particles (A). 
During ageing (B and $\mathrm{C}$ ), adjoining particles partly overcome this stabilization at points of contact, and there is some interdiffusion leading to the formation of "bridges" between particles. In later stages (C), particles are linked by numerous bridges, and the serum solids becomes trapped in small pockets. In the tackified acrylic, the TR is compatible with the serum solids and forms a continuous phase (D). Because the tackifier is also miscible with the acrylic, and because the serum solid phase is no longer able to stabilise the particles, fast interdiffusion at all points of contact between the latex particles can take place (E and F). The role of TR is twofold: it prevents the stabilization of the latex particles by the serum solid phase and it dissolves into the acrylic without being trapped at the particle interfaces.

\section{Conclusions}

It has been discovered that the drying mechanism of an acrylic latex, used in PSAs, is significantly altered by the addition of a waterborne tackifying resin. In the later stages of the drying of the acrylic latex film, water is pinned near the film/air interface, and the water concentration increases with increasing depth into the surface. This drying mechanism enables the surfactant and other water-soluble species in the serum (i.e. the serum solid phase) to be distributed throughout the film depth. Particle coalescence is inhibited by this serum phase, which stabilizes the particles.

When the tackifier is added to the acrylic latex, the particles are able to coalesce because the tackifier forms a continuous phase with the serum solid phase. This phase is miscible with the acrylic, so that diffusion between the latex particles is not inhibited. According to the DMA data, the addition of the tackifier increases the 
polymer's molecular mobility at low frequencies so that polymer reptation during interdiffusion is faster.

Furthermore, it was found that when the TR was in concentrations of 25 wt.\% or more, the latex particles near the air interface coalesce to create a dry skin layer. Water is trapped beneath the skin, and the drying times are increased. Less surfactant can be transported to the interface. The complementary use of AFM and NMR techniques (spectroscopy and profiling) has enabled this understanding to emerge.

The good compatibility between the tackifying resin and the acrylic is apparent in a study of the ageing of tackified PSAs. No phase separation is observed. A decrease in the long component of the $\mathrm{T}_{2}$ relaxation time of the acrylic when blended with the TR likewise indicates that there is mixing at the molecular level, which is consistent with the TR behaving as a solid-like solvent. Current work in our laboratories is determining the interrelationship between film structure, which is influenced by the film formation mechanisms, and adhesive properties. Any trapped water that is caused by skinning during film formation is expected to have a detrimental impact on adhesion.

\section{Acknowledgements}

We dedicate this paper to Hugh Brown in recognition of his inspiring and insightful research. 
Funding for G. Bennett was provided by the Engineering and Physical Sciences

Research Council and by the European Commission. Dr C. Jeynes assisted with the RBS analysis. Dr P. Roose and J. Verstegen assisted with the DMA measurements.

\section{References}

[1] Creton, C., MRS Bull. 28, 434-439, (2003).

[2] Lakrout, H., Sergot, P., and Creton, C., J. Adhesion 69, 307-359 (1999).

[3] Gay C., and Leibler, L., Phys. Rev. Lett. 82, 936-939 (1999).

[4] Toikka G., Spinks G.M., and Brown H.R., Langmuir 17, 6207-6212 (2001).

[5] Zosel, A., Intern. J. Adhesion \& Adhesives 18, 265-271 (1998).

[6] Satas, D., Handbook of Pressure Sensitive Adhesives, (Van Nostrand Reinhold, New York, 1989) 3rd ed.

[7] Shull, K.R., Creton C., J. Pol. Sci. Pt. B. Pol. Phys. 42, 4023-4043 (2004).

[8] Wood, T.G., Adhes. Age 40(7), 19-23 (1987).

[9] Cronin, M.J., Adhes. Age 41(2), 12-16 (1998).

[10] Paiva, A., Sheller, N., Foster, M.D., Crosby, A.J., and Shull, K.R.

Macromolecules 34, 2269-2276(2001).

[11] Comyn, J., Int. J. Adhesion \& Adhesives 15, 9-14 (1995).

[12] Mitchell, R.D. Adhes. Age May 44(5), 29- 49 (2001).

[13] Nakajima, N., Babrowicz, R., and Harrell,E.R., J. Polym. Sci., Polym. Phys. Ed. 44, 1437-1456 (1992).

[14] Aubrey, D. W., and Sherriff, M., J. Polym. Sci., Polym. Chem. Ed. 16, 26312643 (1978).

[15] Aubrey, D. W., and Sherriff, M., J. Polym. Sci., Polym. Chem. Ed. 18, 2597-2608 (1980). 
[16] Sherriff, M., Knibbs, R. W., and Langley, P. G. J. Appl. Polym. Sci. 17, 34233438 (1973).

[17] Vondruska, B.J., Bathina, H.B., Weber C.D., and Pulliam, L.J., Adhes. Age 40(5), 28-32 (1997).

[18] Akiyama, S., Kobori, Y., Sugisaki, A., Koyama, T., and Akiba, I., Polymer, 41, 4021-4027 (2000).

[19] Min, K.E., Kim, H.G., Choi, K.Y., and Kwak, K.H., Polymer - Korea 24, 469476 (2000).

[20] Ryu, D.Y., and Kim, J.K., Polymer 41, 5207-5218 (2000).

[21] Kim, J.K., Ryu, D.Y., and Lee, K.-H., Polymer 41, 5195-5205 (2000).

[22] Takemoto, M.; Karasawa, T; Mizumachi, H., and Kajiyama, M., J. Adhesion 72, 85-96 (2000).

[23] Kim, H.-J., Hayashi, S., and Mizumachi, H., J. Appl. Polym. Sci. 69, 581-587 (1998).

[24] Hayashi, S., Kim, H.-J., Kajiyama, M., Ono, H., and Mizumachi, H., J. Appl. Polym. Sci. 71, 651-663 (1999).

[25] Jovanovic, R., and Dube, M.A., J. Macromol. Sci.-Polym. Rev. C44, 1-51 (2004). [26] Mulvihill, J., Toussaint, A., and De Wilde, M., Prog. Org. Coat. 30, 127-139 (1997).

[27] Charmeau, J.Y., Gerin, P.A., Vovelle, L., Schirrer, R., and Holl, Y., J. Adhesion Sci. Technol. 13, 203-215 (1999).

[28] Zosel, A., and Schuler, B., J. Adhesion 70, 179-195 (1999).

[29] Gerin, P.A., Grohens, Y., Schirrer, R., and Holl, Y., J. Adhesion Sci. Technol. 13, 217-236 (1999).

[30] Tobing, S.D., and Klein, A., J. Appl. Polym. Sci. 79, 2230-2241 (2001). 
[31] Charmeau, J.Y., Berthet, R., Grigreau, C, Holl, Y, and Kientz, E., Int. J. Adhes Adhesives 17, 169-176 (1997).

[32] Mallégol, J., Dupont, O., and Keddie, J.L., Langmuir 17, $7022-7031$ (2001).

[33] Mallégol, J., Gorce, J.-P., Dupont, O., Jeynes, C., McDonald, P.J., and Keddie, J.L., Langmuir 18, 4478-4487 (2002).

[34] Mallégol, J., Dupont, O., and Keddie, J.L., J. Adhes. Sci. Techn. 17, 243-259 (2003).

[35] Milker, R., J. Adv. Mater. 33, 57-62 (2001).

[36] Tobing, S.D., Klein, A., J. Appl. Polym. Sci. 76, 1965-1976 (2000).

[37] Guigner, D, Fischer, C., and Holl, Y., Langmuir 17, 3598-3606 (2001).

[38] Gorce, J.-P., McDonald, P.J., and Keddie, J.L. Eur. Phys. J. E 8, 421-429 (2002).

[39] Chen, X., Davies, M.C., Roberts, C.J., Tendler, S.J.B., Williams, P.M., Davies, J., Dawkes, A.C., and Edwards, J.C., Ultramicroscopy 75, 171-181 (1998).

[40] Glover, P. M., Aptaker, P. S., Bowler, J. R., Ciampi, E. and McDonald, P. J., J. Magn. Reson. 139, 90-97 (1999).

[41] Bennett, G., Gorce, J.-P., Keddie, J.L., McDonald, P.J., and Berglind, H., Magn. Res. Imag. 21, 235-241 (2003).

[42] McDonald, P. J. and Newling, B., Rep. Prog. Phys. 61, 1441-1493 (1998).

[43] Paiva, A., Foster, M.D., and von Meerwall, E.D., J. Polym. Sci.: Pt. B: Polym. Phys. 36, 373-381 (1998).

[44] Keddie, J.L., Mater. Sci. Eng. Rep. R21, 101-170 (1997).

[45] Juhué, D. and Lang, J., Macromolecules 27, 695-701 (1994).

[46] Routh, A. F. and Russel, W. B., Langmuir 15, 7762-7773 (1999). 
[47] Wallin, M., Glover, P. M., Hellgren, A. C., Keddie, J. L., and McDonald, P. J., Macromolecules 33, 8443-8452 (2000).

[48] Aramendia, E., Mallégol, J., Jeynes, C., Barandiaran, M.J., Keddie, J.L., and Asua, J.M., Langmuir 19, 3212-21 (2003). 
Published in Journal of Adhesion (2006) 82(3): pp 217-238

Table 1. Calculated Drying Times for PSA Films

\section{TR Concentration}

(wt.\%)

0

10

25

50

75

100
Film Drying Time ${ }^{\mathrm{a}}$

(min.)

220

280

570

570

1060

1680

${ }^{\mathrm{a}}$ For a valid comparison, experimental values of drying time are adjusted to correspond to the same film thickness $(150 \mu \mathrm{m})$, assuming that the evaporation rate is independent of film thickness. 


\section{Figure Captions}

Figure 1. Dynamic mechanical analysis in tensile mode. Temperature dependence of (a) the tensile storage modulus and (b) $\tan \delta\left(E^{\prime \prime} / E^{\prime}\right)$ measured for neat PSA (dotted lines) and for formulations containing $25 \%$ TR by weight (solid lines). Data from materials from two different batches are shown for each type of PSA. Measurement errors were evaluated through three replicate measurements on one formulated sample. Error bars show the calculated $95 \%$ confidence intervals.

Figure 2. AFM height images of the surface a tackifying resin film at room temperature $\left(\mathrm{ca} .23^{\circ} \mathrm{C}\right.$ ) as a function of the drying time: (a) $20 \mathrm{~min}$.; (b) $60 \mathrm{~min}$; (c) $120 \min .$.

Figure 3. Height (left) and phase (right) AFM images of the top surface of films cast from acrylic/TR blends: (a) neat acrylic latex; (b) 5 wt.\% TR; (c) 10 wt.\% TR; (d) 25 wt.\% TR; (e) and 50 wt.\% TR. Images were obtained within one hour of casting. All image areas are $5 \mu \mathrm{m} \times 5 \mu \mathrm{m}$; height scale $=50 \mathrm{~nm}$ and phase scale $=90$ degrees.

Figure 4. NMR magnetization decay curves constructed from the FID and CPMG experiments for acrylic PSA with varying concentrations of TR, as indicated.

Figure 5. Evolution of the FID obtained from a bilayer of dry TR and acrylic latex after various contact times: (B) 20 min.; (C) 6 hr.; (D) 30 hr. and (E) 260 hr. For comparison, the predicted FID for an equal mixture of acrylic and TR, assuming no 
interaction between them, is shown (A). The experimental FID for a dried layer of a 1:1 blend (by weight) of acrylic latex and TR is also shown $(\mathrm{F})$.

Figure 6. MR profiles obtained over time from a drying acrylic latex film. The time after film casting is stated in the legend. Two stages of drying are shown: (a) the first stage in which the thickness of the layer decreases at a constant rate as evaporation of the water takes place; and (b) the second stage in which the rate of thickness decrease slows down and a non-uniform water distribution develops. The final profile (218 min.) is attributed to the $200 \mu \mathrm{m}$ thick, fully dry acrylic film.

Figure 7. MR profiles obtained during the drying of acrylic PSAs at various concentrations of TR: (a) Neat acrylic latex; (b) 10 wt.\% TR, (c) 25 wt.\% TR, (d) 50 wt.\% TR, (e) 75 wt.\% TR, and (f) neat TR dispersion. Only the second stage of drying is presented for each. The drying times are stated in the legends.

Figure 8. AFM images (height images at the left and phase images on the right) showing the changes at PSA surfaces after ageing at $25{ }^{\circ} \mathrm{C}$ in $45 \%$ relative humidity. Images show neat acrylic latex (a-c) at various times after casting: (a) $2 \mathrm{hr}$; (b) three weeks; (c) seven weeks; and acrylic with 1 wt.\% TR at (d) 2 hr.; (e) one week; and (f) three weeks. All image size are $3 \mu \mathrm{m}$ x $3 \mu \mathrm{m}$; height scale $=40 \mathrm{~nm}$; phase scale $=50$ degrees.

Figure 9. A schematic diagram to illustrate the changes in morphology during the ageing of acrylic latex films (A to C) and tackified acrylic latex (D to F). See the text for a discussion. 
Published in Journal of Adhesion (2006) 82(3): pp 217-238
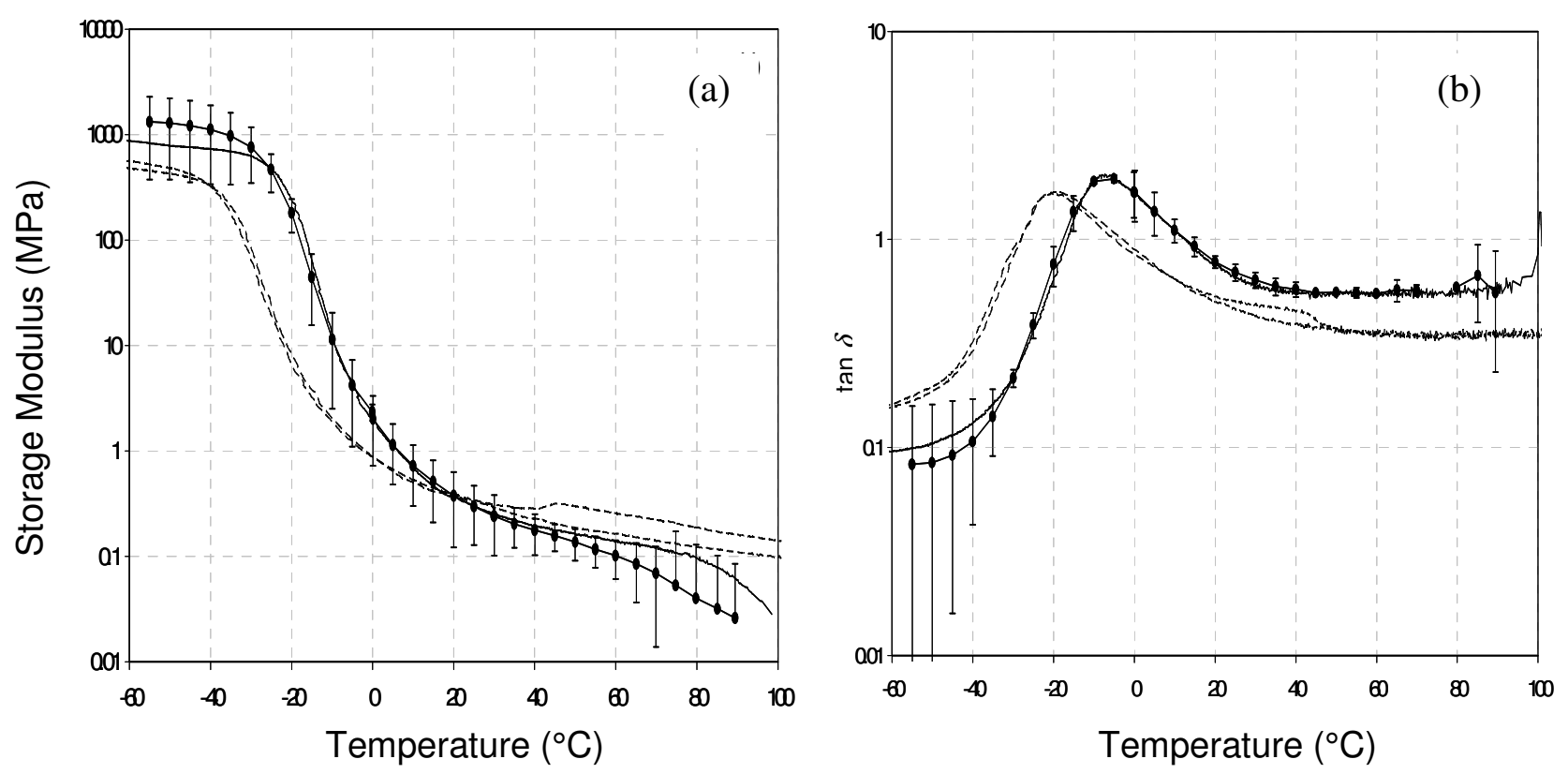

Figure 1 

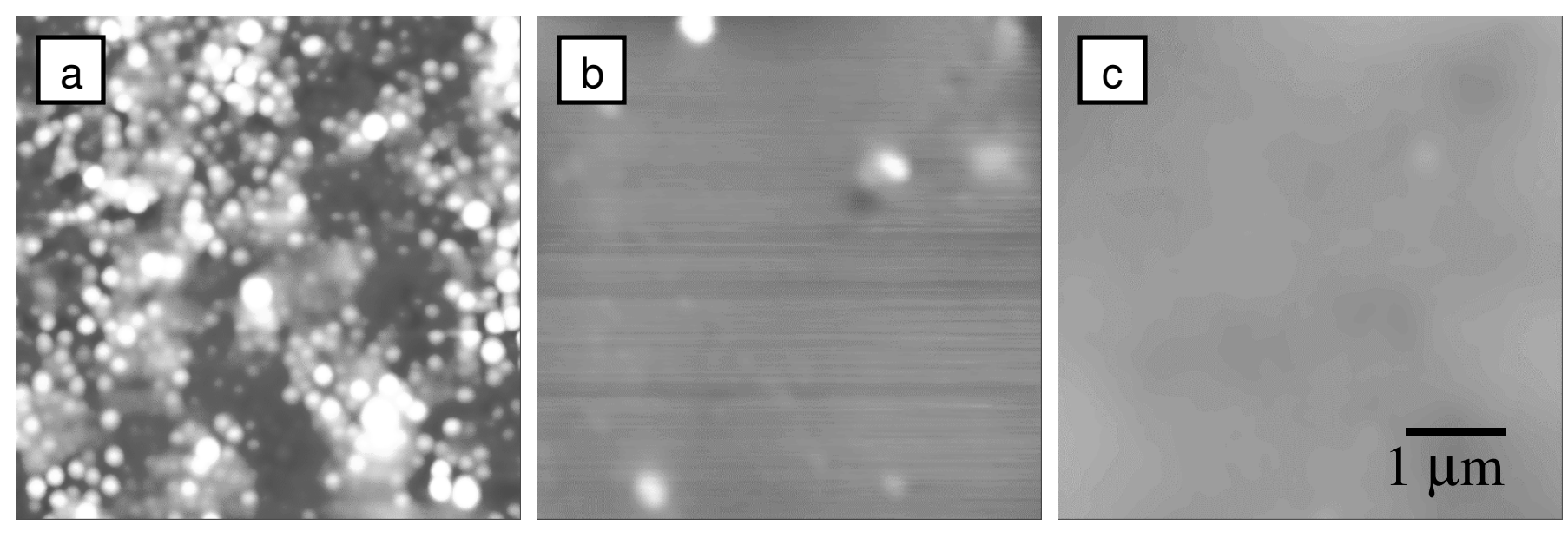

Figure 2 
Published in Journal of Adhesion (2006) 82(3): pp 217-238

Figure 3
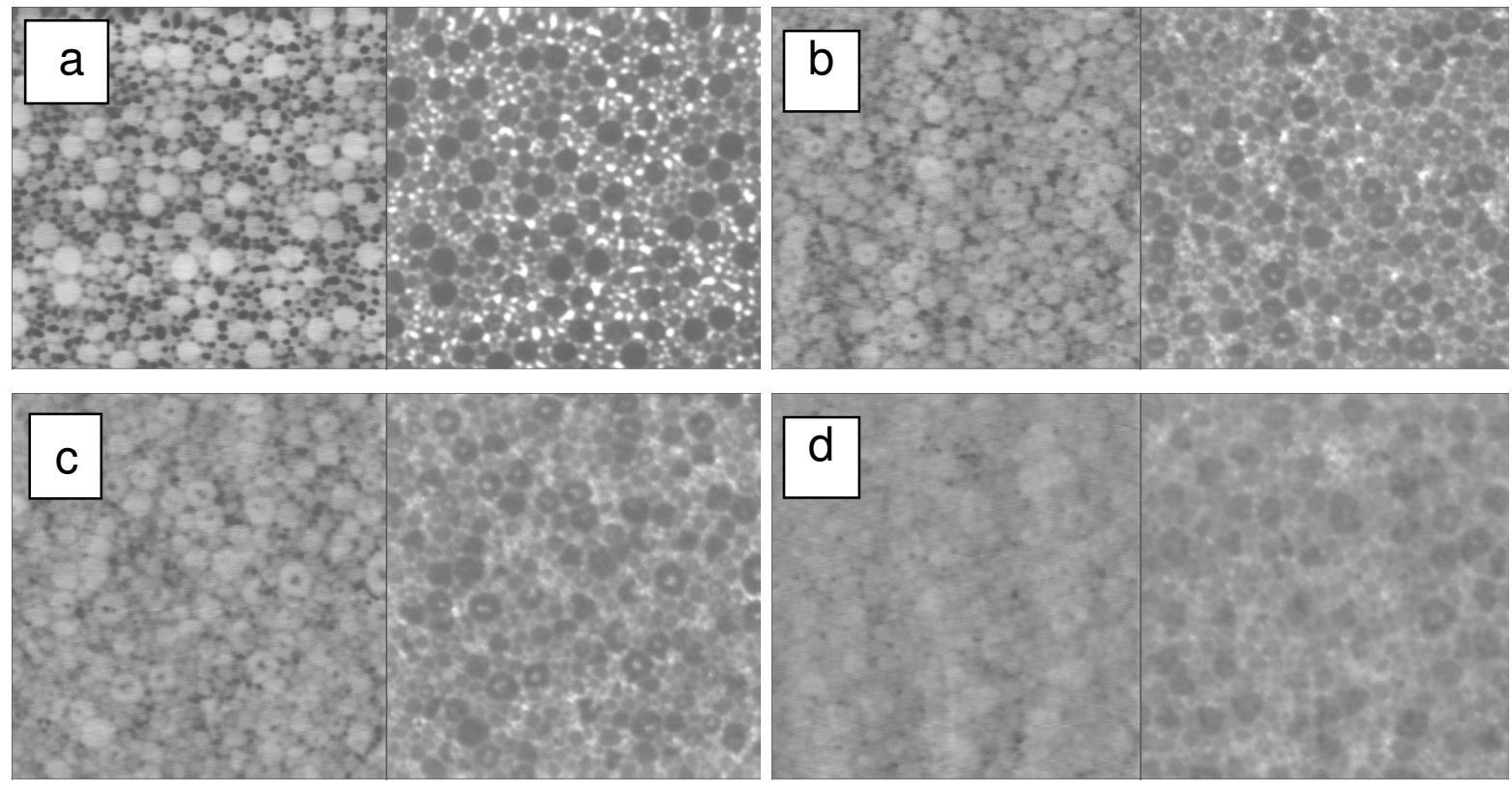

d

e 
Published in Journal of Adhesion (2006) 82(3): pp 217-238

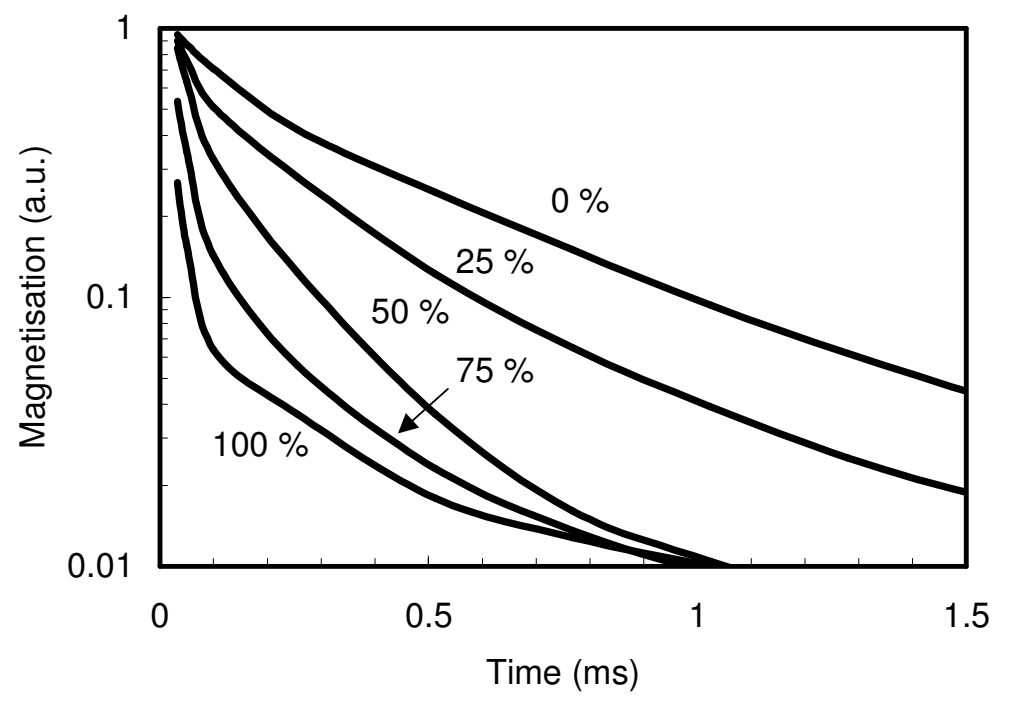

Figure 4 
Published in Journal of Adhesion (2006) 82(3): pp 217-238

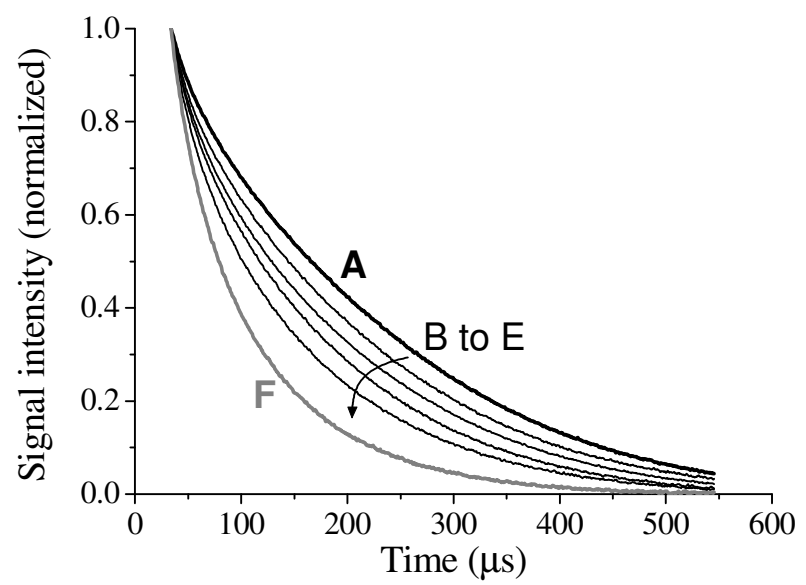

Figure 5 
Published in Journal of Adhesion (2006) 82(3): pp 217-238

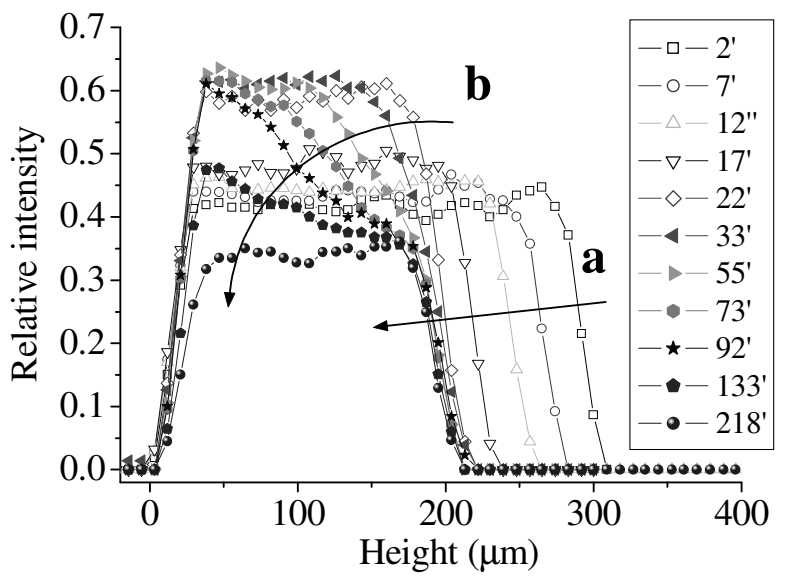

Figure 6 

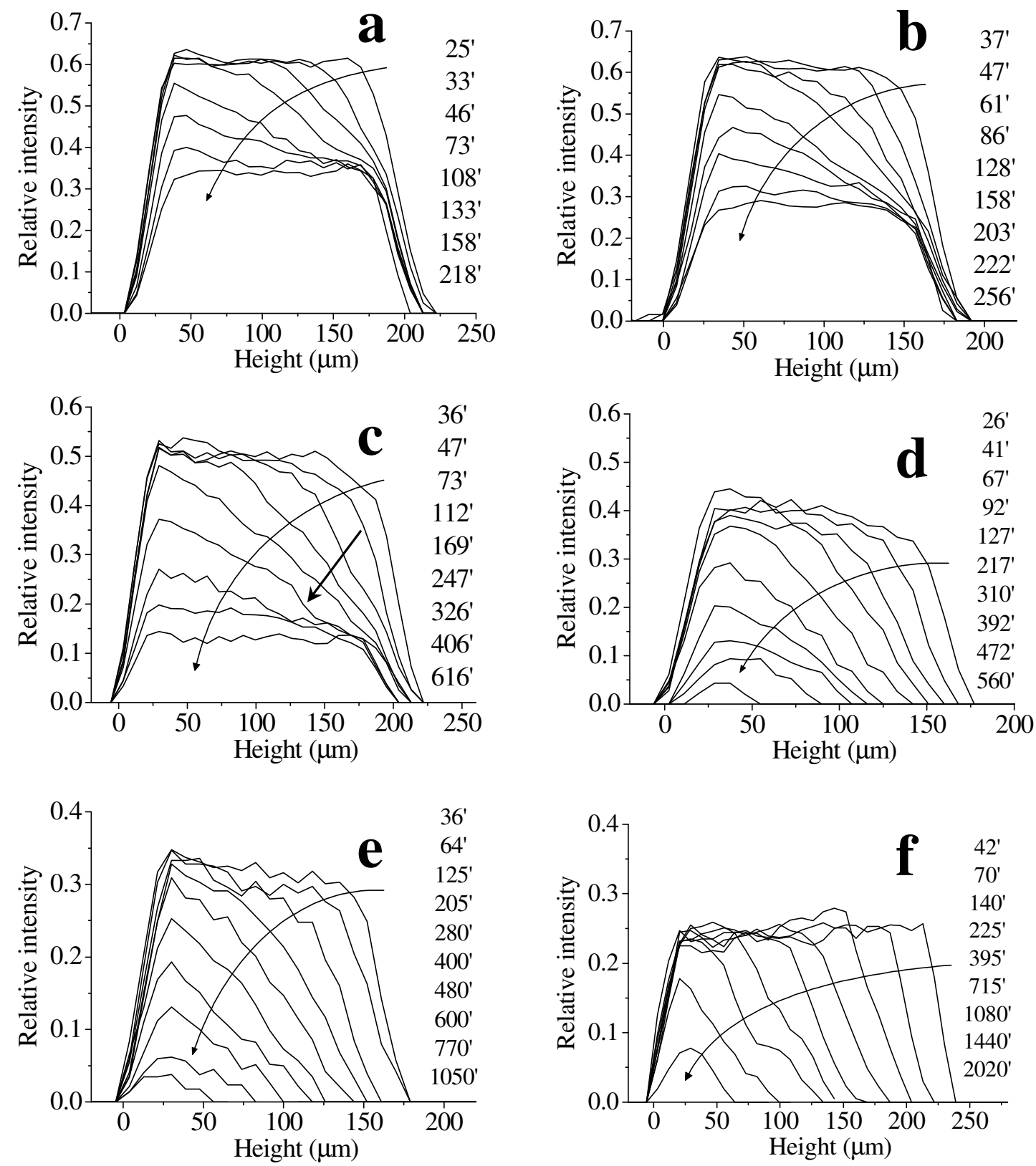

Figure 7 

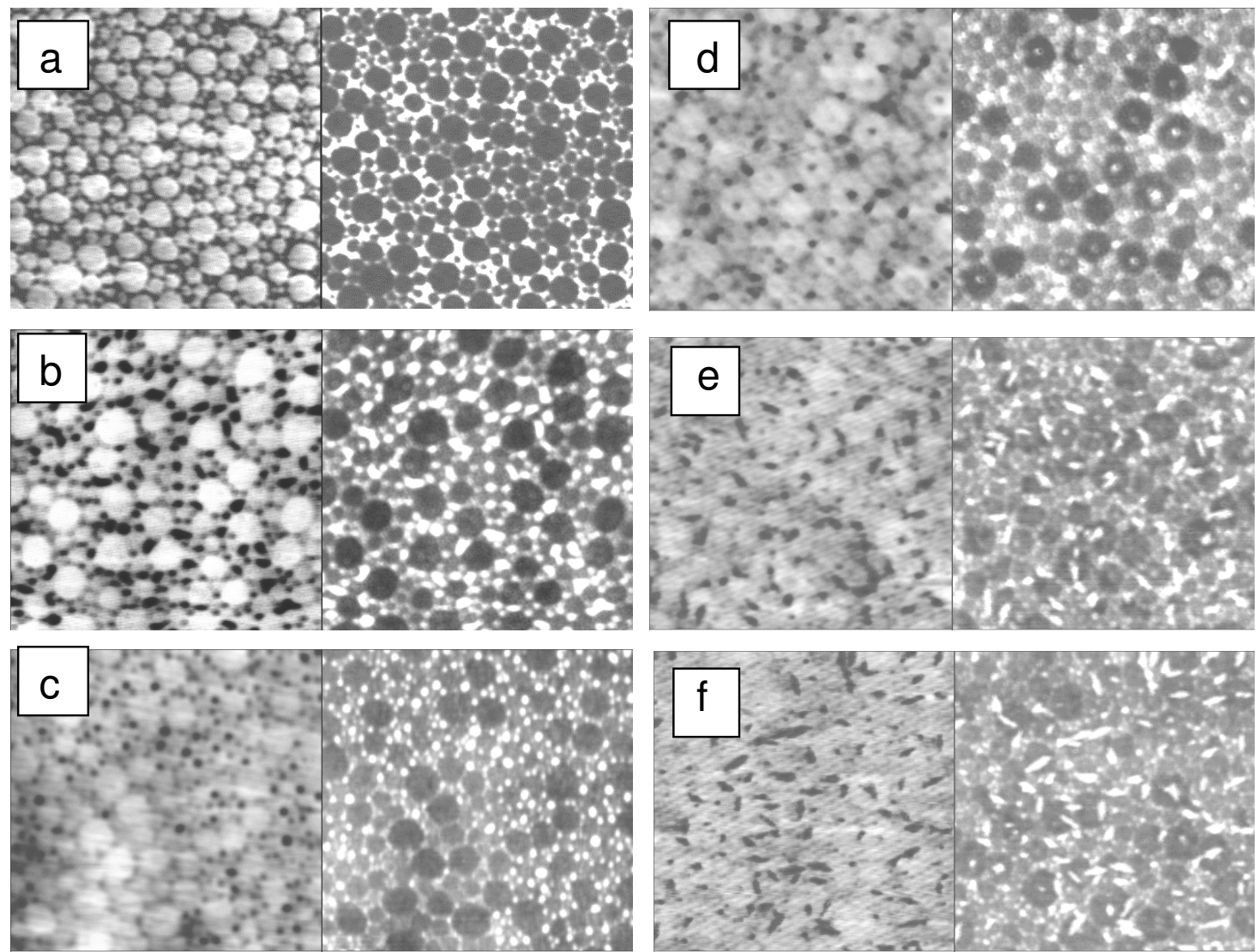

Figure 8 


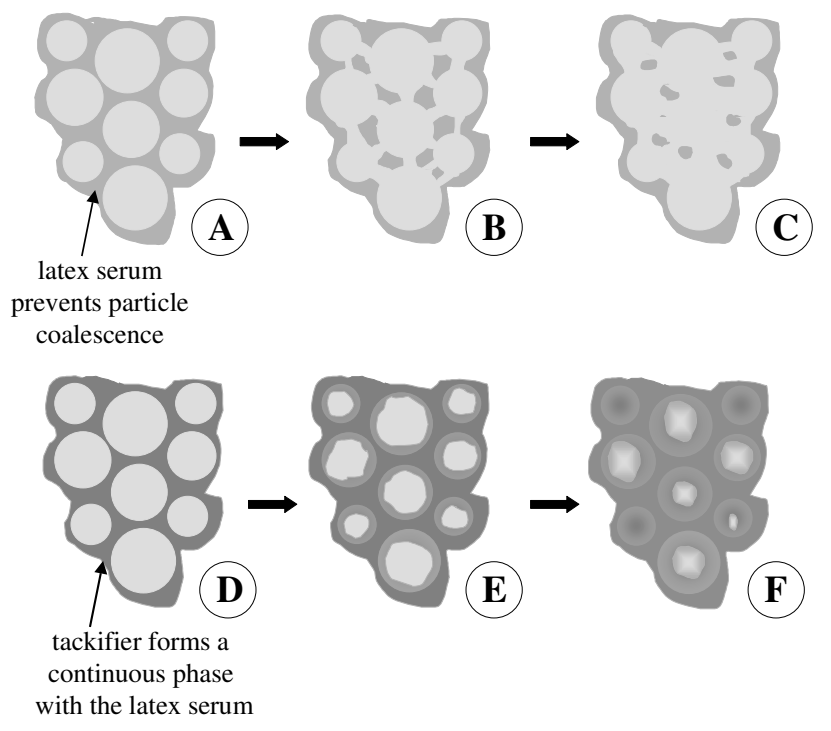

Figure 9 